\title{
Some Guidelines for Publishing Quantitative Research in Sex Roles
}

\author{
Cirleen DeBlaere $^{1} \cdot$ Matthew Hesson-McInnis $^{2}$
}

Published online: 4 December 2015

(C) Springer Science+Business Media New York 2015

Many of the studies published in Sex Roles: A Journal of Research represent quantitative research. To ensure that this research continues to be of high caliber and impact, the Editorial leadership outlines here some general guidelines for consideration by authors who intend to submit research that utilizes quantitative methods and analyses to Sex Roles. Both authors will be part of the journal's editorial team and will serve as Consultants to the Editor for submissions that draw on quantitative methods.

We recognize that quantitative methods are diverse and consistently evolving. Furthermore, methodology and analyses should not be circumscribed by regulations, but rather should be guided by a study's purpose, hypotheses, and data. Given those caveats, the following recommendations are offered as general guidelines, not dictates. These guidelines, however, do reflect good or best practices and should be incorporated where and when appropriate.

\section{Recommendations for the Abstract}

The abstract should highlight the key aspects of the study, including sample size and composition, methods (e.g., survey, online recruitment), analyses (e.g., "A $t$-test indicated that women reported higher levels of gender discrimination than

Cirleen DeBlaere

cdeblaere@gsu.edu

1 College of Education and Human Development, Georgia State University, P.O. Box 3980, Atlanta, GA 30302-3980, USA

2 Department of Psychology, Illinois State University, Normal, IL, USA men"), main results, and some discussion of the study's implications.

\section{Recommendations for the Introduction}

It is important to provide a strong rationale for the study's purpose and hypothesis(es). Consequently, all aspects of a research question should be addressed in the Introduction section. For instance, if a study proposes an indirect effect between an independent variable (X) and dependent variable (Y), through variable $\mathrm{M}$, authors should present theory and relevant prior research to support the hypothesized relations for all variables included in the model. Hypotheses should include clear statements about anticipated relations and, when possible, the direction of those associations. For example, such hypotheses will predict the group with the higher mean score, the positive or negative direction of a correlation, or the ways in which a moderator operates to qualify the relation between an independent variable and dependent variable. Simply stating that a relationship or difference between variables will be found is insufficient, except for the circumstance in which analyses are exploratory and no prior theory or research is available to guide hypotheses.

\section{Recommendations for the Methods}

- Authors should provide sufficient demographic information so as to inform accurately the generalizability of the study's findings (e.g., race/ethnicity, gender, sexual orientation, social class indicators).

- When presenting participant data, authors should discuss missing data. The amount of missing data, as well as whether data are missing at random and how missing data 
were addressed (e.g., listwise deletion, data imputation), should be articulated. If imputed values were calculated, specific details about how values were imputed should be included (e.g., "Full-information maximum likelihood imputation was used to replace $\mathrm{x} \%$ of data points").

- In describing the measures utilized in an investigation, authors should provide relevant reliability and validity data when available. Moreover, when possible, reliability and validity data obtained with samples similar to a study's participants is recommended. Scoring procedures and direction of scores should also be consistently reported (e.g., "Scores were averaged and higher scores indicated higher levels of a given construct").

- In selecting an analytic framework, authors should not cite standard, traditional methods, the use of which is not controversial. In employing newer methods or applying extant methods in novel ways, citations should be included to allow readers to develop a greater understanding of those methods or a novel application of methods. The particular data analysis software package should only be cited for procedures that are not implemented in widely available statistics packages (e.g., SAS, SPSS) or for procedures for which different algorithms may yield somewhat discrepant results (e.g., structural equation modeling).

- Enough detail should be included about how the analyses were implemented so that a statistically proficient reader could replicate the findings.

\section{Recommendations for the Results}

- In presenting the study's results, it is generally helpful for the authors to report findings in a manner that corresponds to the presentation of the hypotheses. The same organizing principles used to present the hypotheses should be followed for the results where possible. Alternatively, authors may wish to consider reorganizing the hypotheses to streamline presentation of the results and to make the connections between the hypotheses, analyses, results, and conclusions clear and consistent.

- Prior to reporting the results, authors should discuss whether the assumptions for the analyses utilized were satisfied. For instance, prior to reporting findings of a structural equation model, it is recommended that authors report whether assumptions of sample size and univariate and multivariate normality were met.

- The criterion for the appropriate sample size for the analytical plan to be executed is useful to discuss and may be determined by articulated best practice guidelines (with a Citation provided) or power analyses. Small samples sometimes can be justified for difficult-to-recruit participants, important research questions, and in some instances when significant and unambiguous effects are obtained, but underpowered analyses are unacceptable.

- Tables and figures should be used as necessary to allow readers to visualize and understand the details of the findings, especially when including the necessary details in textual form would be lengthy and redundant. Tables and figures should not be used when a few sentences could present the same information without loss of clarity. Similar to the presentation of results, Tables should be organized so that the information conveyed in a table parallels the text to which it is connected. Additionally, tables often convey more information, and they do so more efficiently, in print than do figures and thus are preferred.

- Enough detail should be presented in the results so that readers can join the authors in reaching their conclusions or formulate an informed basis for disagreeing with those conclusions.

- Generally, means should be reported along with their accompanying standard deviations (and ranges where informative).

- Exact $p$-values should be reported, even for nonsignificant effects, along with appropriate indicators of effect size for all results (e.g., $\eta p^{2}, R^{2}$ ). Except in a few extenuating circumstances, authors should avoid interpreting non-significant effects $(p \geq .05)$ even if they "approach significance."

\section{Recommendations for the Discussion}

- The discussion should provide a careful interpretation of the findings, and authors should avoid recapitulating the findings and refrain from over-interpreting the results. No new data should be introduced in the Discussion section.

- Implications of the findings should be addressed in terms of further developing relevant theories and when there are clear practical implications (e.g., social policy).

- Broad speculation should be avoided, and any speculative statements should be clearly identified as such. Ideally, speculation should be tied to suggestions for how future research could investigate and ascertain the veracity of those postulations.

- The discussion should clearly articulate the limitations associated with the methods, analyses, and generalizability of the results.

- Suggestions for future research may be addressed, especially when the research process revealed methodological or pragmatic pitfalls that could be avoided in future research. 